\title{
Intelligent Reflecting Surface: Practical Phase Shift Model and Beamforming Optimization
}

\author{
Samith Abeywickrama*†, Rui Zhang*, and Chau Yuen ${ }^{\dagger}$ \\ ${ }^{*}$ National University of Singapore, Singapore \\ ${ }^{\dagger}$ Singapore University of Technology and Design, Singapore \\ Email: samith@u.nus.edu,elezhang@nus.edu.sg, yuenchau@sutd.edu.sg
}

\begin{abstract}
Intelligent reflecting surface (IRS) that enables the control of the wireless propagation environment has been looked upon as a promising technology for boosting the spectrum and energy efficiency in future wireless communication systems. Prior works on IRS are mainly based on the ideal phase shift model assuming the full signal reflection by each of the elements regardless of its phase shift, which, however, is practically difficult to realize. In contrast, we propose in this paper a practical phase shift model that captures the phase-dependent amplitude variation in the element-wise reflection coefficient. Applying this new model to an IRS-aided wireless system, we formulate a problem to maximize its achievable rate by jointly optimizing the transmit beamforming and the IRS reflect beamforming. The formulated problem is non-convex and difficult to be optimally solved in general, for which we propose a low-complexity suboptimal solution based on the alternating optimization (AO) technique. Simulation results unveil a substantial performance gain achieved by the joint beamforming optimization based on the proposed phase shift model as compared to the conventional ideal model.

Index Terms-Intelligent reflecting surface, passive array, beamforming optimization, phase shift model.
\end{abstract}

\section{INTRODUCTION}

Intelligent reflecting surface (IRS) assisted wireless communication has recently emerged as a promising solution to enhance the spectrum and energy efficiency for future wireless systems. Specifically, an IRS is able to establish favourable channel responses by controlling the wireless propagation environment through its reconfigurable passive reflecting elements (see e.g. [2]-[5] and the references therein). However, the existing works on IRS mostly assume an ideal phase shift model with full reflection, i.e., unity amplitude at each reflection element regardless of the phase shift, which, however, is practically difficult to realize due to the hardware limitation.

The amplitude response of a typical passive reflecting element is non-uniform with respect to its phase shift. In particular, the amplitude exhibits its minimum value at the zero phase shift, but monotonically increases and asymptotically approaches unity amplitude at the phase shift of $\pi$ or $-\pi$. This is due to the fact that when the phase shift approaches zero, the image currents, i.e., the currents of a virtual source that accounts for the reflection, are in-phase with the reflecting element currents, and thus the electric field and the current flow in the element are enhanced. As a result, the dielectric

A more comprehensive version of this work has been submitted to IEEE Transactions on Communications [1] loss, metallic loss, and ohmic loss increase dramatically, leading to substantial energy loss and hence low reflection amplitude [6]. Furthermore, these losses mainly come from the semiconductor devices, metals, and dielectric substrates used in the IRS, and thus are not avoidable in practice. In fact, this is a long standing problem for reflection-based metasurfaces [7]. In [8], amplifiers are integrated into the reflecting elements to compensate the energy loss, which is not suitable for passive IRS and also practically costly.

In [2] and [4], by assuming the ideal phase shift model, IRS reflection is designed to have the maximum phase alignment between the IRS-reflected and non-IRS-reflected signals at the designated receivers. In contrast, when the amplitude depends on the phase shift at each reflecting element, such an optimal reflection design is not feasible as each phase shift needs to be properly chosen to have a better balance between the amplitude and phase alignment. Therefore, if the IRS reflection is designed for a practical system based on the ideal phase shift model, it inevitably causes certain performance degradation. To the best of the authors' knowledge, the practical phase shift model and corresponding beamforming optimization algorithm design for IRS-aided wireless systems has not been reported in the literature yet.

This thus motivates this paper, where we first propose a practical phase shift model and verify its accuracy with the experimental results reported in literature. Next, based on this model and considering an IRS-aided point-to-point communication system, we formulate a new problem to maximize its achievable rate by jointly optimizing the transmit beamforming and the IRS reflect beamforming. As this problem is nonconvex, we propose a low-complexity algorithm to solve it sub-optimally by leveraging the alternating optimization (AO) technique. Simulation results are also presented to demonstrate the performance gain by the joint beamforming optimization based on the proposed practical phase shift model over the conventional ideal model.

Notations: In this paper, scalars are denoted by italic letters, vectors and matrices are denoted by bold-face lower-case and upper-case letters, respectively. For a complex-valued vector $\mathbf{v}$, $\|\mathbf{v}\|, \mathbf{v}^{H}$, and $\operatorname{diag}(\mathbf{v})$ denote its $\ell_{2}$-norm, conjugate transpose, and a diagonal matrix with each diagonal element being the corresponding element in $\mathbf{v}$, respectively. Scalar $v_{i}$ denotes the $i$-th element of vector $\mathbf{v}$. For a square matrix $\mathbf{A}, \mathbf{A}_{n, k}$ denotes 


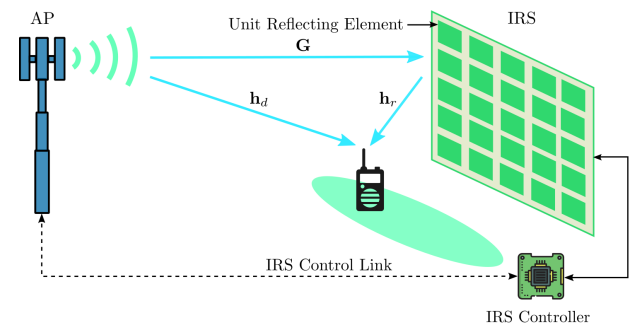

Fig. 1: An IRS-aided wireless system.

its entry in the $n$-th row and $k$-th column. $\mathbb{C}^{x \times y}$ denotes the space of $x \times y$ complex-valued matrices. $j$ denotes the imaginary unit, i.e., $j^{2}=-1$. For a complex-valued scalar $v,|v|, \arg (v)$, and $\bar{v}$ denote its absolute value, phase, and complex conjugate, respectively. $\mathbb{E}(\cdot)$ denotes the statistical expectation.

\section{System ModeL}

We consider a multiple-input single-output (MISO) wireless system where an IRS composed of $N$ reflecting elements is deployed to assist in the communication from an access point (AP) with $M$ antennas to a single-antenna user, as illustrated in Fig. 1. The IRS reflecting elements are programmable via an IRS controller. Furthermore, IRS controller communicates with the AP via a separate wireless link for the AP to control the IRS reflection. It is assumed that the signals that are reflected by the IRS more than once have negligible power due to substantial path loss and thus are ignored. In addition, we consider a quasi-static flat-fading model, where it is assumed that all the wireless channels remain constant over each transmission block. The channels are assumed to be known at the AP by applying, e.g., the channel estimation technique proposed in [9].

Let $\mathbf{h}_{d} \in \mathbb{C}^{M \times 1}, \mathbf{h}_{r} \in \mathbb{C}^{N \times 1}$, and $\mathbf{G} \in \mathbb{C}^{N \times M}$ denote the baseband equivalent channels from the AP to user, from the IRS to user, and from the AP to IRS, respectively. Without loss of generality, let $\mathbf{v} \in \mathbb{C}^{N \times 1}$ denote the reflection coefficient vector of the IRS, where $\left|v_{n}\right| \in[0,1]$ and $\arg \left(v_{n}\right) \in[-\pi, \pi)$ are the amplitude and the phase shift on the combined incident signal, respectively, for $n \in\{1, \ldots, N\}$ [3]. Note that for the ideal phase shift model considered in [2]-[4], $\left|v_{n}\right|=1, \forall n$, regardless of the phase shift, $\arg \left(v_{n}\right)$. The transmit signal at the AP is given by $\mathbf{x}=\mathbf{w} s$, where $\mathbf{w} \in \mathbb{C}^{M \times 1}$ denotes the beamforming vector and $s$ denotes the transmit symbol, which is independent of $\mathbf{w}$, and has zero-mean and unit variance (i.e., $\mathbb{E}\left(|s|^{2}\right)=1$ ). We have dropped the time index for notational simplicity. The received baseband signal at the user is thus given by

$$
y=\left(\mathbf{v}^{H} \mathbf{\Phi}+\mathbf{h}_{d}^{H}\right) \mathbf{x}+z,
$$

where $\mathbf{\Phi}=\operatorname{diag}\left(\mathbf{h}_{r}^{H}\right) \mathbf{G}$ and $z$ denotes the additive white Gaussian noise (AWGN) at the receiver with zero mean and variance $\sigma^{2}$.

In this paper, we aim to maximize the achievable rate or spectrum efficiency (SE) in bits per second per Hertz (bps/Hz)

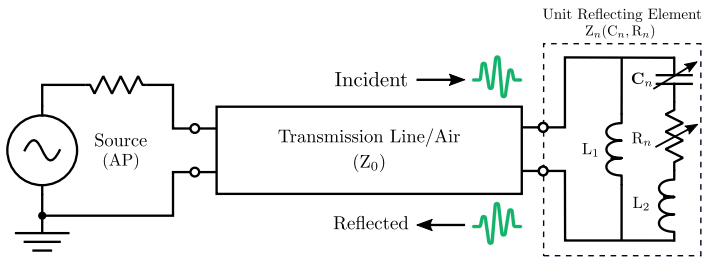

Fig. 2: Transmission line model of a unit reflecting element.

by jointly optimizing the AP beamforming vector $\mathbf{w}$ and the IRS reflection vector $\mathbf{v}$. Accordingly, the achievable rate/SE is given by 1

$$
R_{\mathrm{SE}}=\log _{2}\left(1+\frac{\left|\left(\mathbf{v}^{H} \mathbf{\Phi}+\mathbf{h}_{d}^{H}\right) \mathbf{w}\right|^{2}}{\sigma^{2}}\right) .
$$

\section{Practical Phase Shift Model}

\section{A. Equivalent Circuit Model}

An IRS is typically constructed as a printed circuit board (PCB), where the reflecting elements are equally spaced in a two-dimensional plane. A unit reflecting element is composed of a metal patch on the top layer of the PCB dielectric substrate and a full metal sheet on the bottom layer [3]. Moreover, a semiconductor device 2 , which can vary the impedance of the reflecting element by controlling its biasing voltage, is embedded into the top layer metal patch so that the element response can be dynamically tuned in real time without changing the geometrical parameters [12]. In other words, when the geometrical parameters are fixed, the semiconductor device controls the phase shift and amplitude (absorption level).

As the physical length of a unit reflecting element is usually smaller than the wavelength of the desired incident signal, its response can be accurately described by an equivalent lumped circuit model regardless of the particular geometry of the element [13]. As such, the metallic parts in the reflecting element can be modeled as inductors as the high-frequency current flowing on it produces a quasi-static magnetic field. In Fig. 2, the equivalent model for the $n$-th reflecting element is illustrated as a parallel resonant circuit and its impedance is given by

$$
Z_{n}\left(C_{n}, R_{n}\right)=\frac{j \omega L_{1}\left(j \omega L_{2}+\frac{1}{j \omega C_{n}}+R_{n}\right)}{j \omega L_{1}+\left(j \omega L_{2}+\frac{1}{j \omega C_{n}}+R_{n}\right)},
$$

where $L_{1}, L_{2}, C_{n}, R_{n}$, and $\omega$ denote the bottom layer inductance, top layer inductance, effective capacitance, effective resistance, and angular frequency of the incident signal, respectively. Note that $R_{n}$ determines the amount of power dissipation due to the losses in the semiconductor devices, metals, and dielectrics, which cannot be zero in practice, and

\footnotetext{
${ }^{1}$ Note that the considered system model can be also applied to wireless power transfer (WPT) [3] as the harvested radio-frequency (RF) energy at the receiver is generally modeled as an increasing function of the received signal power [10], i.e., the term $\left|\left(\mathbf{v}^{H} \mathbf{\Phi}+\mathbf{h}_{d}^{H}\right) \mathbf{w}\right|^{2}$ given in [2].

${ }^{2}$ In practice, a positive-intrinsic-negative (PIN) diode, a variable capacitance (varactor) diode, or a metal-oxide-semiconductor field-effect transistor (MOSFET) can be used as the semiconductor device mentioned here [7], [11, [12].
} 


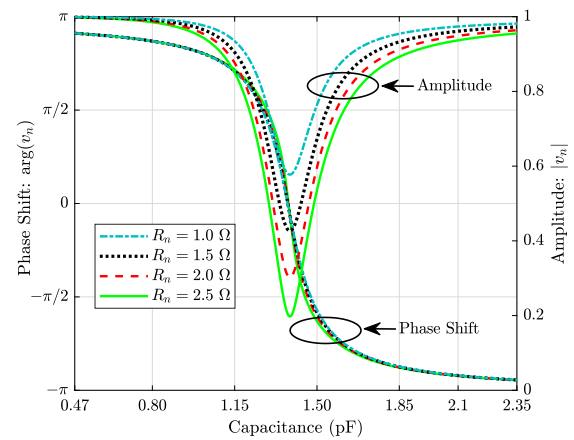

(a) Phase shift and amplitude versus $C_{n}$ and $R_{n}$.

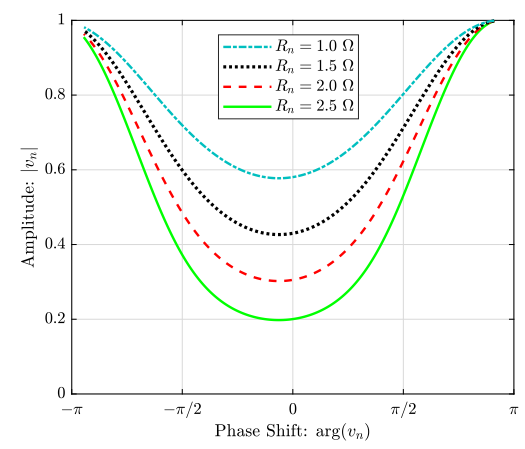

(b) Amplitude versus phase shift.

Fig. 3: Reflection coefficient of a unit reflecting element.

$C_{n}$ specifies the charge accumulation related to the element geometry and semiconductor device. As the transmission line diagram in Fig. 2 depicts, the reflection coefficient, i.e., $v_{n}$ in (1), is the parameter that describes the fraction of the reflected electromagnetic wave due to the impedance discontinuity between the free space impedance $Z_{0}$ and element impedance $Z_{n}\left(C_{n}, R_{n}\right)$ [14], which is given by

$$
v_{n}=\frac{Z_{n}\left(C_{n}, R_{n}\right)-Z_{0}}{Z_{n}\left(C_{n}, R_{n}\right)+Z_{0}} .
$$

Since $v_{n}$ is a function of $C_{n}$ and $R_{n}$, the reflected electromagnetic waves can be manipulated in a controllable and programmable manner by varying $C_{n}$ 's and $R_{n}$ 's.

To demonstrate this, Fig. 3 illustrates the behaviour of the amplitude and the phase shift, i.e., $\left|v_{n}\right|$ and $\arg \left(v_{n}\right)$, respectively, for different values of $C_{n}$ and $R_{n}$. Note that to align with the experimental results in [7], $C_{n}$ is varied from $0.47 \mathrm{pF}$ to $2.35 \mathrm{pF}$ when $L_{1}=2.5 \mathrm{nH}, L_{2}=0.7 \mathrm{nH}, Z_{0}=377 \Omega$, and $\omega=2 \pi \times 2.4 \times 10^{9}$. It is observed that a reflecting element is capable of achieving almost $2 \pi$ full phase tuning, while the phase shift and amplitude both vary with $C_{n}$ and $R_{n}$ in general. It is also observed that the minimum amplitude occurs near zero phase shift and approaches unity (the maximum) at the phase shift of $\pi$ or $-\pi$, which is explained as follows. When the phase shift is around $\pi$ or $-\pi$, the reflective currents (also termed as image currents) are out-of-phase with the element currents, and thus the electric field and the current

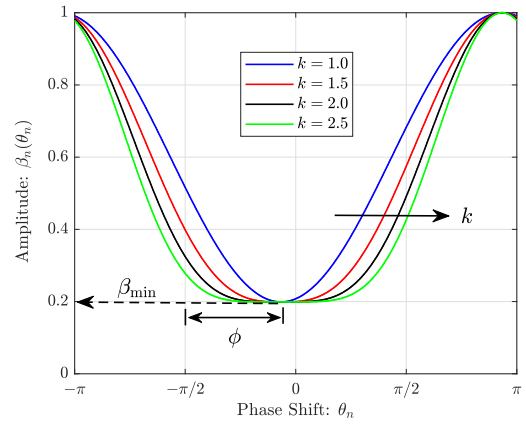

(a) The phase shift model with different parameters.

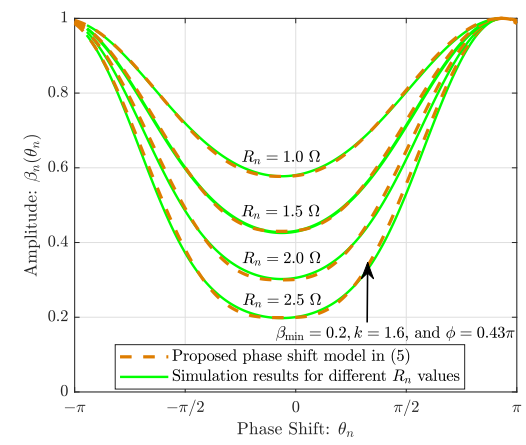

(b) Simulation results for the proposed phase shift model.

Fig. 4: The proposed phase shift model.

flow in the element are both diminished, thus resulting in minimum energy loss and highest reflection amplitude. In contrast, when the phase shift is around zero, the reflective currents are in-phase with the element currents, and thus the electric field and the current flow in the element are both enhanced. As a result, the dielectric loss, metallic loss, and ohmic loss increase dramatically, leading to substantial energy dissipation and thus lowest reflection amplitude. Furthermore, it is worth noting that the numerical results illustrated in Fig. 3 are in accordance with the experimental results reported in literature (see [6] and Fig. 5 (b) in [7]), indicating that the circuit model given by (3) and (4) accurately captures the physics of a reflecting element in practice.

It is also worth noting that to obtain an ideal phase shift control, where $\left|v_{n}\right|=1, \forall \arg \left(v_{n}\right) \in[-\pi, \pi)$, each reflecting element should exhibit zero energy dissipation. However, for practical hardware, energy dissipation is unavoidable 3 and the typical behaviour of the reflection amplitude is similar to Fig. 3 Therefore, incorporating a practical phase shift model to design beamforming algorithms is essential to optimize the performance of IRS-aided wireless systems.

${ }^{3}$ In [7], $R_{n}=2.5 \Omega$ in each reflecting element due to the diode junction resistance, while in [6], although the reflecting element does not contain any semiconductor device, its amplitude response follows a similar shape to Fig. 3 due to the metallic loss and dielectric loss. 


\section{B. Proposed Phase Shift Model}

In order to characterize the fundamental relationship between the reflection amplitude and phase shift for designing IRS-aided wireless systems, we propose in this subsection an analytical model for the phase shift which is generally applicable to a variety of semiconductor devices used for implementing the IRS. Let $v_{n}=\beta_{n}\left(\theta_{n}\right) e^{j \theta_{n}}$ with $\theta_{n} \in$ $[-\pi, \pi)$ and $\beta_{n}\left(\theta_{n}\right) \in[0,1]$ respectively denote the phase shift and the corresponding amplitude. Specifically, $\beta_{n}\left(\theta_{n}\right)$ can be expressed as

$$
\beta_{n}\left(\theta_{n}\right)=\left(1-\beta_{\min }\right)\left(\frac{\sin \left(\theta_{n}-\phi\right)+1}{2}\right)^{k}+\beta_{\min },
$$

where $\beta_{\min } \geq 0, \phi \geq 0$, and $k \geq 0$ are the constants related to the specific circuit implementation. As depicted in Fig. 4(a), $\beta_{\min }$ is the minimum amplitude, $\phi$ is the horizontal distance between $-\pi / 2$ and $\beta_{\min }$, and $k$ controls the steepness of the function curve. Note that for $k=0$, (5) is equivalent to the ideal phase shift model, i.e., $\beta_{n}\left(\theta_{n}\right)=1, \forall n$. In practice, IRS circuits are fixed once they are fabricated and these parameters can be easily found by a standard curve fitting tool.

Fig. 4 (b) illustrates that the proposed phase shift model closely matches the simulation results presented in Section III-A for a practical reflecting element. In the sequel, we adopt the model in (5) for beamforming design in IRS-aided wireless communication. Moreover, we assume that the circuits of the reflecting elements are all identical, and thus the same model parameters, i.e., $\beta_{\min }, \phi$, and $k$, apply to each of the elements.

\section{BEAMFORMING OPTIMIZATION}

\section{A. Problem Formulation}

We aim to jointly optimize $\mathbf{w}$ and $\mathbf{v}$ such that the achievable rate, $R_{\mathrm{SE}}$ given in (2), is maximized. The problem is formulated as

$$
\begin{aligned}
(\mathrm{P} 0): \max _{\mathbf{w}, \mathbf{v},\left\{\theta_{n}\right\}} & \left|\left(\mathbf{v}^{H} \mathbf{\Phi}+\mathbf{h}_{d}^{H}\right) \mathbf{w}\right|^{2} \\
\text { s.t. } & \|\mathbf{w}\|_{2}^{2} \leq P_{T}, \\
& v_{n}=\beta_{n}\left(\theta_{n}\right) e^{j \theta_{n}}, \forall n=1, \ldots, N, \\
& -\pi \leq \theta_{n} \leq \pi, \forall n=1, \ldots, N,
\end{aligned}
$$

where $P_{T}$ denotes the maximum transmit power constraint at the AP. For any given phase shift $\mathbf{v}$, it is known that the maximum-ratio transmission (MRT) is the optimal transmit beamforming solution to (P1), i.e., $\mathbf{w}^{*}=\sqrt{P_{T}} \frac{\left(\mathbf{v}^{H} \mathbf{\Phi}+\mathbf{h}_{d}^{H}\right)^{H}}{\left\|\left(\mathbf{v}^{H} \mathbf{\Phi}+\mathbf{h}_{d}^{H}\right)\right\|}$. By substituting $\mathrm{w}^{*}$ to $(\mathrm{P} 0)$, the problem for optimizing the IRS reflection is reformulated as

$$
\begin{aligned}
(\mathrm{P} 1): \max _{\mathbf{v},\left\{\theta_{n}\right\}} & \left\|\left(\mathbf{v}^{H} \mathbf{\Phi}+\mathbf{h}_{d}^{H}\right)\right\|^{2} \\
\text { s.t. } & v_{n}=\beta_{n}\left(\theta_{n}\right) e^{j \theta_{n}}, \forall n=1, \ldots, N, \\
& -\pi \leq \theta_{n} \leq \pi, \forall n=1, \ldots, N .
\end{aligned}
$$

Although simplified, problem (P1) is non-convex and difficult to be optimally solved in general. In the next subsection, we solve (P1) by applying the $\mathrm{AO}$ technique.

\section{B. Proposed AO Algorithm}

We propose an $\mathrm{AO}$ algorithm to find an approximate solution to (P1), by iteratively optimizing the phase shift of one of the $N$ reflecting elements with those of the others being fixed at each time, and repeatedly doing this procedure for all $N$ elements until the objective value in (10) converges. The convergence is guaranteed as the optimal value of (P1) is upper-bounded by a finite value. To this end, the problem for optimizing the reflection of the $n$-th element is formulated as

$$
\begin{gathered}
(\mathrm{P} 2): \max _{\theta_{n}} \beta_{n}^{2}\left(\theta_{n}\right) \Psi_{n, n}+\beta_{n}\left(\theta_{n}\right)\left|\varphi_{n}\right| \cos \left(\arg \left(\varphi_{n}\right)-\theta_{n}\right) \\
\text { s.t. }-\pi \leq \theta_{n} \leq \pi,
\end{gathered}
$$

where $\boldsymbol{\Psi}=\operatorname{diag}\left(\mathbf{h}_{r}^{H}\right) \mathbf{G} \mathbf{G}^{H} \operatorname{diag}\left(\mathbf{h}_{r}\right), \hat{\mathbf{h}}_{d}=\operatorname{diag}\left(\mathbf{h}_{r}^{H}\right) \mathbf{G} \mathbf{h}_{d}$, and $\varphi_{n}=\left(\sum_{m \neq n}^{N} \boldsymbol{\Psi}_{n, m} v_{m}\right)+2 \hat{h}_{d, n}$. Note that (13) is obtained by taking the terms associated with $\beta_{n}\left(\theta_{n}\right)$ and $\theta_{n}$ in the expansion of (10), while the derivation is omitted due to the space limitation. The problem (P2) is a single-variable nonconvex optimization problem, for which we propose a closedform approximate solution that can be efficiently computed in the next subsection.

\section{An Approximate Solution to (P2)}

The key to approximately solve (P2) in closed-form lies in re-expressing (13) in a more tractable model. However, an approximate model of a general nonlinear function can only fit the original function locally, which we refer to as the trust region. In our problem, the trust region should essentially be the one that encloses the optimal solution of (P2), denoted by $\theta_{n}^{*}$.

Define $f\left(\theta_{n}\right) \triangleq \beta_{n}^{2}\left(\theta_{n}\right) \boldsymbol{\Psi}_{n, n}+\beta_{n}\left(\theta_{n}\right)\left|\varphi_{n}\right| \cos \left(\arg \left(\varphi_{n}\right)-\right.$ $\left.\theta_{n}\right)$. It is not difficult to observe that for the ideal phase shift model considered in [2]-[4], $\beta_{n}\left(\theta_{n}\right)$ and $\theta_{n}$ can be designed to maximize $f\left(\theta_{n}\right)$ (or (13) ) by setting $\beta_{n}^{*}\left(\theta_{n}\right)=1$ and $\theta_{n}^{*}=$ $\arg \left(\varphi_{n}\right), \forall n$. However, such an optimal reflection design is not feasible for a practical IRS due to the dependency of $\beta_{n}\left(\theta_{n}\right)$ on $\theta_{n}$ as depicted in Fig. 3 (b). For instance, if $\arg \left(\varphi_{n}\right)=0$, $\theta_{n}^{*}=0$ may not be a favourable phase design as it yields the lowest reflection amplitude. In this case, $\theta_{n}^{*}$ needs to be properly chosen to have a better balance between $\beta_{n}\left(\theta_{n}\right)$ and $\arg \left(\varphi_{n}\right)$. In particular, since the minimum $\beta_{n}\left(\theta_{n}\right)$ occurs near zero phase shift and approaches the maximum at $\pi$ and $-\pi$, $\theta_{n}^{*}$ should slightly deviate from $\arg \left(\varphi_{n}\right)$ towards $\pi$ (or $-\pi$ ) when $\arg \left(\varphi_{n}\right)$ is non-negative (negative). The trust region that encloses $\theta_{n}^{*}$ is thus given by

$$
\theta_{n}^{*} \in\left[\arg \left(\varphi_{n}\right),(-1)^{\lambda} \pi\right],
$$

with $\lambda=0$ when $\arg \left(\varphi_{n}\right) \geq 0$ and $\lambda=1$ otherwise.

Motivated by the above result, a high-quality approximate solution to problem (P2) can be obtained numerically via a one-dimensional (1D) search over $\left[\arg \left(\varphi_{n}\right),(-1)^{\lambda} \pi\right]$, which, however, is still computationally inefficient. Alternatively, a closed-form approximate solution can be obtained by fitting a quadratic model through three points over the trust region 


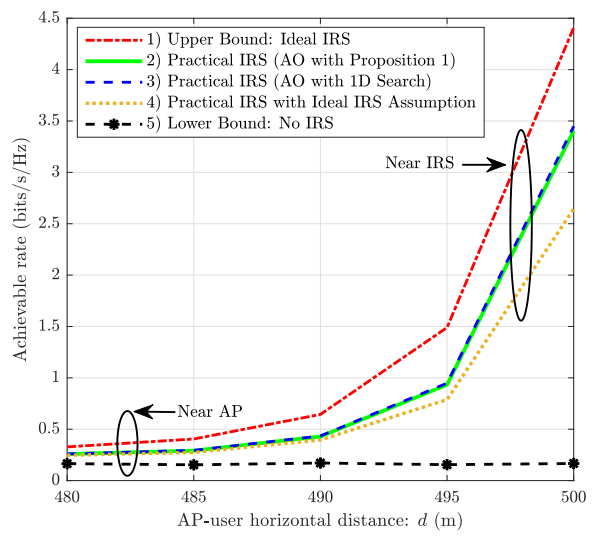

Fig. 5: Achievable rate versus the AP-user horizontal distance when $N=40$.

(which are obtained via equally sampling the trust region), i.e., $\theta_{A}=\arg \left(\varphi_{n}\right), \theta_{B}=\frac{\arg \left(\varphi_{n}\right)+(-1)^{\lambda} \pi}{2}$, and $\theta_{C}=(-1)^{\lambda} \pi$, as given in the following proposition.

Proposition 1: Let $f_{1}=f\left(\theta_{A}\right), f_{2}=f\left(\theta_{B}\right)$, and $f_{3}=$ $f\left(\theta_{C}\right)$. The approximate solution to (P2) obtained by fitting a quadratic curve through the points $\left(\theta_{A}, f_{1}\right),\left(\theta_{B}, f_{2}\right)$, and $\left(\theta_{C}, f_{3}\right)$ is given by

$$
\hat{\theta}_{n}^{*}=\frac{(-1)^{\lambda} \pi\left(3 f_{1}-4 f_{2}+f_{3}\right)+\arg \left(\varphi_{n}\right)\left(f_{1}-4 f_{2}+3 f_{3}\right)}{4\left(f_{1}-2 f_{2}+f_{3}\right)} .
$$

Proof: See Appendix A

It is worth noting that Proposition 1 essentially corresponds to a single iteration of successive quadratic estimation with trust region refinement [15]. The overall iterative algorithm to solve (P1) is given in Algorithm 1.

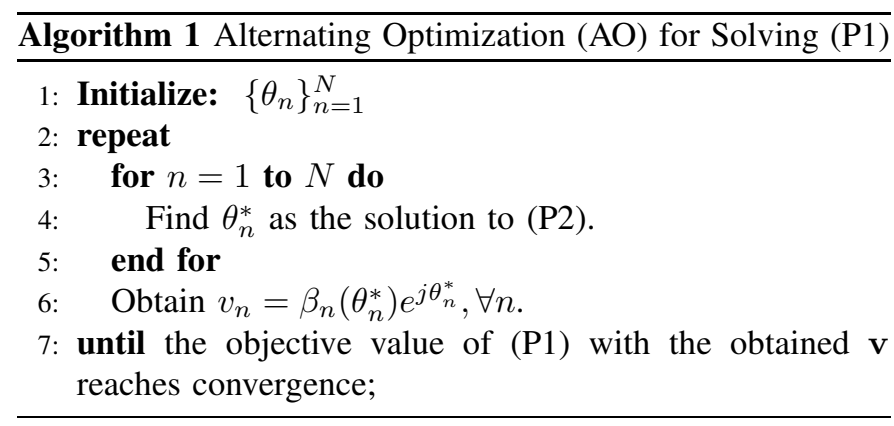

\section{Simulation Results}

We consider a MISO downlink wireless system consisting of an AP with $M=2$ antennas and a single-antenna user. It is assumed that an IRS composed of $N$ reflecting elements is deployed in the vicinity of the user while the AP and IRS are assumed to be located 500 meters (m) apart. Rayleigh fading is assumed for all the channels involved, and the signal attenuation at a reference distance of $1 \mathrm{~m}$ is set as $40 \mathrm{~dB}$. The path loss exponents are set to $2.2,2.8$, and 3.8 for the channels between AP-IRS, IRS-user, and AP-user,

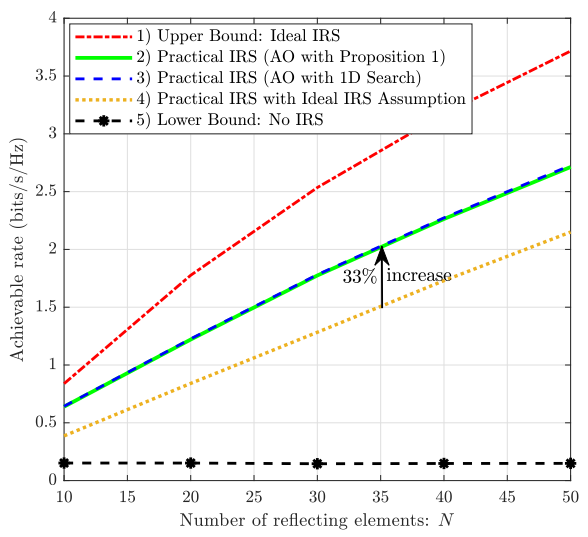

Fig. 6: Achievable rate versus number of reflecting elements at $d=498 \mathrm{~m}$.

respectively, according to [2]. The total transmit power at the $\mathrm{AP}$ is $P_{T}=36 \mathrm{dBm}$ and $\sigma^{2}=-94 \mathrm{dBm}$.

The user is assumed to lie on a horizontal line that is in parallel to that connecting the AP and IRS, with the vertical distance between these two lines equal to $2 \mathrm{~m}$. By varying the horizontal distance between the AP and user, denoted by $d$, in Fig. 5, the achievable rate averaged over 1000 channel realizations is shown for the following schemes:

1) Upper bound: solving the following problem, which assumes the ideal phase shift model, and its solution is given in [2].

$$
\begin{aligned}
(\mathrm{P} 3): \max _{\tilde{\mathbf{v}}} & \left\|\left(\tilde{\mathbf{v}}^{H} \mathbf{\Phi}+\mathbf{h}_{d}^{H}\right)\right\|^{2} \\
\text { s.t. } & \left|\tilde{v}_{n}\right|^{2}=1, \forall n=1, \ldots, N .
\end{aligned}
$$

2) Beamforming optimization by the $\mathrm{AO}$ algorithm under the proposed practical phase shift model with $\beta_{\min }=$ $0.2, k=1.6$, and $\phi=0.43 \pi$, while the problem ( $\mathrm{P} 2)$ is solved using Proposition 1.

3) Beamforming optimization by the $\mathrm{AO}$ algorithm under the proposed practical phase shift model with $\beta_{\min }=$ $0.2, k=1.6$, and $\phi=0.43 \pi$, while the problem ( $\mathrm{P} 2)$ is solved using 1D search.

4) Beamforming optimization assuming the ideal phase shift model [2], while the practical phase shift model is used for computing the achievable rate.

5) Lower bound: the system without using an IRS by setting $\mathbf{w}^{*}=\sqrt{P_{T}} \frac{\mathbf{h}_{d}}{\left\|\mathbf{h}_{d}\right\|}$.

Note that the initial phase shift values of the proposed $\mathrm{AO}$ algorithm, i.e., $\left\{\theta_{n}\right\}_{n=1}^{N}$, are randomly selected from $\{\pi,-\pi\}$ such that each reflecting element has the maximum reflection amplitude.

It is observed from Fig. 5 that 2) performs very close to 3). Proposition 1 thus provides a practically appealing solution to (P2) considering its performance and low complexity. It is also observed that when the user moves closer to the IRS, the performance gap between 2) and 4) increases. This is due to the fact that the user benefits from the stronger reflecting channel via IRS $\left(\mathbf{h}_{r}\right)$, and therefore accurate reflection design 


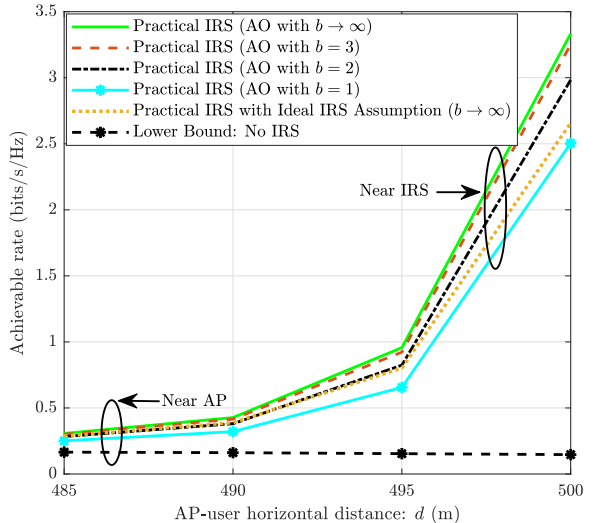

Fig. 7: Achievable rate versus the AP-user horizontal distance when $N=40$ in the case of discrete phase shift.

at the IRS becomes more crucial. In contrast, when the user moves toward the AP, the performance gap between 2) and 4) decreases as the AP-user direct channel $\left(\mathbf{h}_{d}\right)$ becomes dominant and the effect of IRS reflection becomes less significant. Moreover, by fixing the user at $d=498 \mathrm{~m}$ and varying the number of reflecting elements, $N$, in Fig. 6, we plot the average achievable rate. It is also observed that the performance gap between 2) and 4) increases with $N$ as the IRS reflecting channel becomes stronger.

Next, we consider that the phase shift at each element of the IRS can only take a finite number of discrete values, which are equally spaced in $[-\pi, \pi)[16]$. Denote by $b$ the number of bits used to represent each of the levels. Then the set of phase shifts at each element is given by $\mathcal{F}=\{0, \Delta \theta, \ldots, \Delta \theta(K-1)\}$ where $\Delta \theta=2 \pi / K$ and $K=2^{b}$. When the user moves closer to the IRS, in Fig. 7, we compare the average achievable rate for different values of $b$ with the practical and ideal phase shift model. Note that for the finite values of $b$, problem (P2) is solved by performing a $1 \mathrm{D}$ search over $\mathcal{F}$. As expected, the performance increases with $b$. Moreover, it is observed that beamforming optimization under the practical phase shift model with $b=2$ performs even better than that of ideal phase shift model with $b \rightarrow \infty$.

\section{Conclusion}

In this paper, we proposed a practical IRS phase shift model. Based on this new model and considering an IRS-aided MISO system, we formulated and solved a joint transmit and reflect beamforming optimization problem to maximize the achievable rate, by applying the AO technique. Our simulation results validated our proposed analytical model and showed that beamforming optimization based on the conventional ideal phase shift model, which has been widely used in the literature, may lead to significant performance loss as compared to the proposed practical model. In future work, it is worth investigating such performance difference in more general IRS-aided wireless communication setups, such as multi-user systems [2], [4], OFDM-based system [17], physical layer security system [18], simultaneous wireless information and power transfer (SWIPT) systems [19], [20], and so on.
APPENDIX A

BEAMFORMING OPTIMIZATION

\section{A. Proof of Proposition 1}

Given three points $\theta_{A}=\arg \left(\varphi_{n}\right), \theta_{B}=\frac{\arg \left(\varphi_{n}\right)+(-1)^{\lambda} \pi}{2}$, $\theta_{C}=(-1)^{\lambda} \pi$ and their corresponding function values $f_{1}, f_{2}$, $f_{3}$, we seek to determine three constants $a_{0}, a_{1}$, and $a_{2}$ such that the following quadratic function is constructed,

$$
g\left(\theta_{n}\right)=a_{0}+a_{1}\left(\theta_{n}-\theta_{A}\right)+a_{2}\left(\theta_{n}-\theta_{A}\right)\left(\theta_{n}-\theta_{B}\right) .
$$

When $\theta_{n}=\theta_{A}, \theta_{n}=\theta_{B}$, and $\theta_{n}=\theta_{C}$, the constants $a_{0}, a_{1}$, and $a_{2}$ can be respectively obtained. Substituting them into the stationary point of $g\left(\theta_{n}\right)$, i.e., $\hat{\theta}_{n}^{\star}=\frac{\theta_{A}+\theta_{B}}{2}-\frac{a_{1}}{2 a_{2}}$, allows us to obtain (16). The proof is thus completed.

\section{REFERENCES}

[1] S. Abeywickrama, R. Zhang, Q. Wu, and C. Yuen, "Intelligent reflecting surface: Practical phase shift model and beamforming optimization," Submitted to IEEE Trans. Commun., [Online]. Available: https://arxiv.org/abs/2002.10112.

[2] Q. Wu and R. Zhang, "Intelligent reflecting surface enhanced wireless network via joint active and passive beamforming," IEEE Trans. Wireless Commun., vol. 18, no. 11, pp. 5394-5409, Nov. 2019.

[3] Q. Wu and R. Zhang, "Towards smart and reconfigurable environment: Intelligent reflecting surface aided wireless network," IEEE Commun. Mag., vol. 58, no. 1, pp. 106-112, Jan. 2020.

[4] C. Huang, A. Zappone, G. C. Alexandropoulos, M. Debbah, and C. Yuen, "Reconfigurable intelligent surfaces for energy efficiency in wireless communication," IEEE Trans. Wireless Commun., vol. 18, no. 8, pp. 4157-4170, Aug. 2019

[5] E. Basar, M. Di Renzo, J. De Rosny, M. Debbah, M. Alouini, and R. Zhang, "Wireless communications through reconfigurable intelligent surfaces," IEEE Access, vol. 7, pp. 116753-116773, Aug. 2019.

[6] H. Rajagopalan and Y. Rahmat-Samii, "Loss quantification for microstrip reflectarray: Issue of high fields and currents," in Proc. IEEE Antennas and Propag. Society Int. Symposium, Jul. 2008, pp. 1-4.

[7] B. O. Zhu, J. Zhao, and Y. Feng, "Active impedance metasurface with full 360 reflection phase tuning," Scientific reports, vol. 3, pp. 30593064, Oct. 2013.

[8] M. E. Bialkowski, A. W. Robinson, and H. J. Song, "Design, development, and testing of X-band amplifying reflectarrays," IEEE Trans. on Antennas and Propag., vol. 50, no. 8, pp. 1065-1076, Aug. 2002.

[9] B. Zheng and R. Zhang, "Intelligent reflecting surface-enhanced OFDM: Channel estimation and reflection optimization," IEEE Wireless Commun. Lett., DOI:10.1109/LWC.2019.2961357, Dec. 2019.

[10] Y. Zeng, B. Clerckx, and R. Zhang, "Communications and signals design for wireless power transmission," IEEE Trans. Commun., vol. 65, no. 5 , pp. 2264-2290, May 2017.

[11] W. Tang, X. Li, J. Y. Dai, S. Jin, Y. Zeng, Q. Cheng, and T. J. Cui, "Wireless communications with programmable metasurface: Transceiver design and experimental results," [Online]. Available: https://arxiv.org/abs/1811.08119.

[12] F. Liu et al., "Intelligent metasurfaces with continuously tunable local surface impedance for multiple reconfigurable functions," Phys. Rev. Applied, vol. 11, no. 4, pp. 44024-44 033, Apr. 2019.

[13] S. Koziel and L. Leifsson, Surrogate-based modeling and optimization. Springer, 2013.

[14] D. M. Pozar, Microwave Engineering (3th Edition). New York John Wiley \& Sons, 2005.

[15] R. P. Brent, Algorithms for Minimization without Derivatives. PrenticeHall, 1973.

[16] Q. Wu and R. Zhang, "Beamforming optimization for wireless network aided by intelligent reflecting surface with discrete phase shifts," IEEE Trans. Commun., DOI:10.1109/TCOMM.2019.2958916, Dec. 2019.

[17] Y. Yang, B. Zheng, S. Zhang, and R. Zhang, "Intelligent reflecting surface meets OFDM: Protocol design and rate maximization," [Online]. Available: https://arxiv.org/abs/1906.09956. 
[18] M. Cui, G. Zhang, and R. Zhang, "Secure wireless communication via intelligent reflecting surface," IEEE Wireless Commun. Lett., vol. 8 , no. 5, pp. 1410-1414, Oct. 2019.

[19] Q. Wu and R. Zhang, "Joint active and passive beamforming optimization for intelligent reflecting surface assisted SWIPT under QoS constraints," [Online]. Available: https://arxiv.org/abs/1910.06220.

[20] C. Pan, H. Ren, K. Wang, M. Elkashlan, A. Nallanathan, J. Wang, and L. Hanzo, "Intelligent reflecting surface aided MIMO broadcasting for simultaneous wireless information and power transfer," [Online]. Available: https://arxiv.org/abs/1908.04863. 